\title{
A Brief Analysis of the Relationship between Housing, Mental Health and Wellbeing under the Eco-City Context
}

\author{
Li-Li MA \\ School of Economics\& Management, Lanzhou Jiaotong University \\ Lanzhou 730070, P. R. China \\ 13919000080@163.com
}

Key words: Housing, Mental health, Wellbeing, Housing affordability, Social support, Housing policy.

\begin{abstract}
Housing is not only the provision of physical shelter but also a complex source of residents' mental health and wellbeing. Housing type, housing condition and neighborhood quality are directly affect inhabitants' mental health and wellbeing. Suitability, affordability and quality of housing are among the issues that help to determine the experience of housing for households. Additionally, housing is a special kind of place or setting with attributes, features and ambience. It is usually defined by systems of activities which may call attention to certain temporal, psychological, economic, emotional, behavioral features of the inhabitants in the place.
\end{abstract}

\section{Introduction}

It has been recognized that public health is closely related to the built environment from four aspects at different spatial scales, including nature contact, buildings, public spaces and urban form [1]. With the increasingly urban development, constantly changing urban environment not only provide citizens more convenient daily life but also has negative impact on the physical and mental health of inhabitants. Mental health and wellbeing are essential public health priorities during establishing livable and harmonious cities because the rapid expansion of urban agglomerations worldwide often results in deleterious mental-health consequences on inhabitants living in city. Under the circumstances, establishing a more healthy and habitable eco-city is drawing public attention in many countries, especially in developing countries. Based on Roseland' study, mental health and wellbeing of public are critical indicators of eco-city performance [2]. Also it is worth noting that mental health and wellbeing of public are directly and indirectly concerned with decent, affordable, safe, convenient, and economically mixed housing. Accordingly, it is necessary to analyze the relationship between housing, mental health and wellbeing under the eco-city context. Besides, housing policy plays relatively indirect and significant role in improving mental health and wellbeing of inhabitants during creating eco-city. This essay intends to critically analyze the relationship between housing, mental health and well-being. In terms of the direct effect of housing on mental health and wellbeing, characteristics of the housing will be discussed from aspect of house category, housing condition and neighborhood quality. With regard to indirect relationship, many social factors concerning housing tend to be considered including sense of identity and attachment, sense of personal control, housing affordability, and social support. Finally, role of housing policy will be stated to critically evaluate its important effect in ameliorating mental health and well-being of inhabitants in eco-city.

\section{Relationship between Housing, Mental Health and Well-Being}

Shelter is a basic human requirement, and housing is one of the most elementary needs for urban population. Jackson asserts that humanity spend more than $90 \%$ of their lives indoors and "sense of place" is a broadly discussed as a public health construct, and the health impact of housing includes physical, psychological, social, spiritual, and aesthetic effects[3]. Briefly, characteristics of housing can directly affect mental health and wellbeing. Also housing can indirectly impact mental health 
by shifting psychosocial processes with known mental health consequences.

\section{Direct Relationship between Housing, Mental Health and Wellbeing}

\section{The Impact of Housing Category on Mental Health and Wellbeing}

Housing type, namely physical structure, could directly influence mental health and wellbeing of residents to some extent. According to Evans, Wells \& Moch, high-rise, multiple dwelling units are detrimental to the psychological well-being of mothers with young children and young children themselves [4]. Psychological distress and social interaction deficiency could occur due to social isolation of mothers as well as limited play opportunities for children. Specifically, resources and facilities in many high-rise buildings are insufficient for residents or are located relatively far from residences. This can possibly generate more loneliness and diminished territorial control of inhabitants, especially for women, compared with the people who have similar backgrounds and live in other types of housing. Moreover, parents living in large multiple-dwelling units usually deal with the scarcity of play spaces nearby through keeping their children inside apartments. For instance, many amenities such as lobbies, lounges, and other small-group spaces are lacking or located comparatively far from residences. It would bring about insufficient residential control and feelings of ownership and heighten conflict between generations. Additionally, living high-rise or multiple dwelling units remove a principal avenue for parents to know about their neighbors. With regard to children, such constraints also minimize their play and communication opportunities with others, and it plays adverse role in terms of children's physical and psychological development.

\section{The Impact of Housing Condition on Mental Health and Wellbeing}

Housing quality is another important factor posing direct effect on occupants' mental health and wellbeing. Evans, Wells, \& Moch (2003) present some explanations for the possible association between problems with housing quality and mental health, including difficulties with repairs and landlords, insecurity or tenure concerns, repeated relocations, less controllable social interactions, and shame associated with poor housing [4]. To large scale, European housing and health research by the World Health Organization is a cross-sectional study which has indicated that there is a closer relationship existing between living in dwelling and depression or anxiety [1]. Inhabitants are apt to suffer from anxiety and depression because dwelling usually has inadequate protection against outside aggression such as cold, noise, drought, and has little space for solitude or freedom. Other factors generated from housing also, to some extent, jeopardize wellbeing of residents; including deficient light or external view, fear of losing dwelling due to high property management fee, incapacity to move due to financial restrictions and a pessimistic image of the neighborhood. These factors do not facilitate the socialization. Additionally, housing quality characteristically includes some aspect of structural quality such as building material and furnishings, maintenance and upkeep such as cleaning agents that minimize indoor emissions, as well as amenities such as effective ventilation system, central heat and hot water temperature regulators. Evans et al. (2000) assert that psychological well-being can affect one's judgment about dwelling environment quality [5]. For example, people, whose housing condition is lower than others, are often complaining and suffering psychological distress. When people move into better housing, their mental health improves compared with those who do not move. There are substantial representative evidences of associations between housing quality and psychological distress or mental health. Evans, Wells, \& Moch (2003) argue that people in housing which has poor structural quality, dampness, or lack of plumbing, in all probability, have been found to be suffer from depression, anxiety, and other symbols of psychological distress[4]. Thus, poor-quality housing cannot meet the residential satisfaction, and it could attribute some of their predicament to themselves. Particularly among parents in poor housing, they are more likely to be anxious about potential safety hazards including unsatisfactory safety protection, numerous of housing code violations and close contiguity to higher volume street traffic, all of which may results in childhood injury rates and threaten to children's physical and psychological health. 


\section{The Impact of Neighborhood Quality on Mental Health and Wellbeing}

With regard to the location aspects of housing, the neighborhood in which one lives may directly and indirectly influence health outcomes. Neighborhood quality is related to social cohesion, sense of trust and collective efficacy. A feature of neighbourhood is not only a function of physical qualities but also a product of risks and opportunities, a nature of the social organization and interaction, a relationship between political, as well as economic and cultural aspects. Besides, neighbourhood may also have indirect impact on the health of inhabitants by influencing health risk behaviours such as smoking, drug and alcohol use, diet and nutrition. These factors can by turns affect health and prove that unstable neighborhood can seriously play a negative role in quality of life improvement. When people move to new house, they usually change the neighbourhood as well. A research, which is about evaluations of three experiments with housing voucher programs for low-income families in the United States, indicates that moving from low-income neighborhoods to middle income communities is associated with boosted mental health for both adults and children [6]. Besides, neighborhood with poor social functions is associated with poor mental health and wellbeing among the inhabitants. For instance, residents often concern accessibility to infrastructure, transport, shopping center, kindergartens and open spaces as well as proximity to services, facilities in the neighborhood. In other words, mental health and wellbeing also be negatively influenced by neighborhood featured by a relative lacking availability of services such as health and educational services, recreational possibilities and other facilities, features of natural and built environment as well as qualitatively poor social networks.

\section{Indirect Relationship between Housing, Mental Health and Wellbeing}

Housing is not only a physical shelter but also a significant health resource, especially mental health and wellbeing resource. It should be recognized that there are direct, namely "hard", physical or material ways in which housing can influence mental health and wellbeing. It is also noted that there are indirect, namely "soft", social and meaningful ways that may account for how the housing affect mental health and wellbeing. Four psychosocial processes affected by the housing are sense of identity and attachment, housing affordability, personal control, and social support.

\section{Sense of Identity and Attachment Provided By Housing}

Dupuis \& Thorns (1998) present a concept which is housing can provide “ontological security”, defined as a sense of confidence, trust and reliability in the world [7]. A sense of stability and continuity could be provided through home ownership and family ownership because home is a source of personal control and privacy as well as a site of steadiness in the material and social environment. Indeed, according to Dunn (2002), owner-occupiers are more likely to report better self-rated and wellbeing status than renters. His Other studies, especially in Britain found that, even after other key socio-economic variables were considered, people in rented dwellings, mostly those in the public-rental property, have higher death rates than people in owner-occupied households [8]. This is, in some degree, due to the extent of security and control that homeowners have been in relation to renters. Home ownership is also recognized to provide a secure sense of home, and the feeling is undoubtedly crucial to wellbeing. Home owners are capable of reforming and modifying their dwellings which ultimately enhances their perception of home. Such helplessness generated from repossession and relocation make contribution to apparent insecurity among renters which can then make them expose to stress and stress-related illnesses. Besides, home is not only a space for daily routine and rituals for children but its ownership is a rite of passage, source of pride and achievement, which creates part of an adult identity. Additionally, home can enhance an individual's sense of control over his or her life since it is a refuge that offers independence from the surveillance of the contemporary world. Thus, home is correlated with metal health and wellbeing because it can provide a foundation for place attachment and identity.

\section{The Impact of Housing Affordability on Mental Health and Wellbeing}

Affordable housing can be generally defined as "housing of an adequate basic standard that 
provides reasonable access to work opportunities and community services and that is available at a cost which does not cause substantial hardship to the occupants"[9]. A deficiency of affordable housing has negative impact on the competitiveness and efficiency of the urban economy plus the maintenance of social cohesion. Many figures indicate that the number of families who are suffering from some form of housing stress is growing [9]. It can be shown that the health effects can be important through research on the impacts of housing payment issues on health. For instance, renters and recent purchasers are suffer from constant stress related to a lack of money, which can result in family members' anxiety and pressure on family relationships. Furthermore, financial hardship outcomes, including children missing out on school activities and adequate health care, are generated from low purchasing power and high price of housing. Under the circumstances, housing affordability issues are another component of the multiple shortcomings that can adversely affect health and wellbeing. Especially for low-income people, housing affordability, in addition to other stressors, can be a cause of independent continuing stress. According to Jackson, JB, the tension and anxiety associated with a lack of perpetual, affordable housing may lead to child neglect, with children then becoming depressed, aggressive or hard for parents to cultivate [3]. From a social point of view, these consequences could make contribution to social instability due to shortage of equal right, which would threaten social harmony and sustainability.

\section{Sense of Personal Control Generated From Housing}

Disney (2006) asserts that people feel better and have improved mental health when they can control their surroundings. Sense of helplessness may occur when opportunities for control over the environment are frustrated. He also holds that helplessness is performed through exposing subject to an uncontrollable stimulus like noise [9]. Building can make contribution to helplessness. For instance, university students who are living in dormitories with long corridor designs usually experience more different degree of helplessness compared with those living in suite arrangement. This is because uncontrollable social interaction makes adverse impact on students' mental health and wellbeing. Similarly, people living in high-rise housing and poor-quality housing have feeling of helplessness associated with more uncontrollable social interaction. Additionally, acute noise, several crowding and malodorous pollutants could make residents lack sense of personal control and then suffer from helplessness. Accordingly, many design features of housing should be focused on through providing an array of social interaction spaces from small private space for solitude to larger open space for more public interaction opportunities. These factors benefit to creating greater perceived control and comfort in residential settings. Moreover, housing with large structures, lack of small group spaces and poor visual monitor capability is to the disadvantage of territorial control and sense of ownership. It is associated with both real crime and anxiety of crime. Besides, favorable neighboring environment of housing also plays an important role in influencing residents' sense of personal control. For example, public housing inhabitants who relocate to middle-class suburban neighborhoods have more feeling of mastery increase than other public housing residents who move to low-income neighboring environment [10].

\section{A Potential Link between Housing and Mental Health and Wellbeing: Social Support}

It has been extensively recognized that social factors make contribution to influencing the mental health and wellbeing of population. Combination of social support, social interaction, as well as sense of social cohesion is the foundation of the more current emphasis and building social capital. Also it has been identified to correlate positively with mental health and wellbeing. Specific to housing issues, firstly, many housing literatures show that an excessively large number of the defectively housed are members of most vulnerable groups of society, including ethnic minority groups, female-headed households and people who are marginalized in contemporary urban sprawl [11]. Thus, it is reasonable to deduce that housing brings a significant anxiety and pressure on households, especially on disadvantaged low-income households. Additional stress would impair their wellbeing. Accordingly, there are potential associations between housing, social support and psychological distress, and the adverse impact of housing would be moderated by insights of social support resources. Secondly, built environment around housing could affect the development and 
maintenance of socially supportive networks. For instance, the possibility of social interaction is more in community where entrances to residential units are adjacent, proximate or are directly connected to main pedestrian paths or meeting spaces. In the third place, the phenomenon of social withdrawal and socially supportive relationships impairment usually occurred due to more crowded residential settings such as higher number of people per room. Especially for children, study indicates that more conflict and less responsive parenting often appear during parent-child interaction in more crowded homes [5]. Fourthly, communication is undoubtedly a component of social support. It could be interfered with some irritability and negative affect generated from indoor environment of housing. Taking noise as an example, it can lead to the expression of aggression, and residents exposed to noise are usually less likely to communicate with each other or help others. This could prove that either noise or pollution exposure of housing increase adverse effect, touchiness, even aggression. These factors, to some extent generate negative impact on communication and social support.

\section{Role of Housing Policy during Eco-City Establishment}

In consideration of direct and indirect relationship between housing, mental health and wellbeing of public, creating favorable housing conditions, relevant ancillary facilities and satisfactory neighborhood is considered to be important for improving mental health and wellbeing of public. In order to achieve social harmony during eco-city establishment, housing policy planning should be focused on, and it plays a significant role in adjustment of housing quality, quantity, location, cost, price, and housing rights. The principles of housing policy planning should be identified during eco-city establishment. Based on national housing policy norms, housing policy in eco-city should have independence and exemplary effect. Specifically, the first target of the housing policy planning is ought to create a comfortable indoor environment, improve the living quality, provide amicable outdoor environment and then enhance the sustainability and habitability of dwelling environment. Besides, the housing policy planning in eco-city should aim to adjust to market demand for housing and cater to development of regional economy because housing is one of the main essential factors which promote industry development. Also, during housing policy planning, housing can be recognized an approach for attracting talented people by means of improving housing condition and promulgating a series of share purchase policies. Multi-level housing supply structure should be provided to satisfy different needs of people with different income level. In other words, the scheme could provide different types of housing to ensure that household with low or moderate income may live in eco-city. As a whole, some factors are ought to be recognized including adequate physical housing, social supportive features of neighborhoods, protection from violence, protecting against toxicity and human development, mental health and the built environment. Consequently, housing policy in eco-city establishment should be based on sustainability a criterion, which is 'meeting the needs of the present without compromising the ability of future generations to meet their own needs' [12].

\section{Conclusion}

In conclusion, it is evident that housing is not only the provision of physical shelter but also a complex source of residents' mental health and wellbeing. Housing type, housing condition and neighborhood quality are directly affect inhabitants' mental health and wellbeing. There are also existing relationships between housing, mental health and wellbeing from aspect of identity and attachment, sense of personal control, housing affordability, and social support. Suitability, affordability and quality of housing are among the issues that help to determine the experience of housing for households. Additionally, housing is a special kind of place or setting with attributes, features and ambience. It is usually defined by systems of activities which may call attention to certain temporal, psychological, economic, emotional, behavioral features of the inhabitants in the place. Also, housing has both internal and external significance, which may be referred to as its psychosocial and socio-spatial relevance. Specially, the impacts of housing on mental health and 
wellbeing in developing societies could be more severe due to the mostly rapid urbanization, declining socioeconomic conditions, and increasing inequalities. Thus, housing policy dealing with issues with respect to housing stress should be focused on because it can make considerable contributions to making ecological and sustainable city become reality. Some strategies for implementations could play crucial role in creating high-performance of city and catering the residents' expectation for more sustainable and habitable city. Within such a comprehensive approach, the state should recognize the interrelationship between housing and health, asserting that housing is not only a physical shelter, but a significant health resource. Therefore, it is needed to appeal a greater co-operation among government ministries and agencies on housing and health. It is to the benefits of wide-ranging housing and health policies improvement and then establishing harmonious society.

\section{Acknowledgement}

This research was financially supported by Gansu Social Science Planning Project (number: YB071) and Gansu Science and Technology Agency Soft Science Project (number: 1504ZKCA017-3).

\section{References}

[1] Gibson, M, Petticrew, M, Bambra, C, Sowden, AJ, Wright, KE, \&Whitehead, M 2011, 'Housing and health inequalities: A synthesis of systematic reviews of interventions aimed at different pathways linking housing and health’, Journal of Health \& Place. 17(1)175-184,

[2] Roseland, M 1997, 'Dimensions of eco-city ', Journal of cities. 14(4)197-202

[3] Jackson, JB 1995, ‘A Sense of Place, a Sense of Time’, Journal of Design Quarterly. (164)24-27

[4] Evans, GW, Wells, NM \& Moch, A 2003, 'Housing and mental health: a review of the evidence and a methodological and conceptual critique’, Journal of Social Issues. 59(3)475-500

[5] Evans GW, Wells NM, Chan E \& Saltzman H 2000, 'Housing quality and mental health', Journal of Consulting and Clinical Psychology. 68(3)526-530

[6] Johnson MP, Ladd HF \& Ludwig J 2002, 'The benefits and costs of residential mobility programs for the poor', Journal of Housing Studies. 17(1)125-138

[7] Dupuis, A \& Thorns, D 1998, 'Home, home ownership and the search for ontological security', Journal of Sociological Review. 46(1)25-47

[8] Dunn, JR 2000, 'Housing and health inequalities: review and prospects for research', Journal of Housing Studies. 15(3) 341-366

[9] Disney, J. 2006, 'Over our heads: Housing costs \& Australian families', Journal of Australian Quarterly.78 (2) 4-11

[10] Rosenbaum JE, Reynolds L \& Deluca S 2002, 'How do places matter? The geography of opportunity, self-efficacy, and a look inside the black box of residential mobility’, Journal of Housing Studies. 17(1)71-82

[11] Thorns DC.1990, 'The production of homelessness: from individual failure to system inadequacies', Journal of Housing Studies. 4(4)253-266

[12] Choguill CL. 2007, 'The search for policies to support sustainable housing', Journal Habitat International. 31(1)143-149 